\title{
Sur le problème de Cauchy et les problèmes de Fourier pour les équations paraboliques dans un domaine non borné
}

\author{
par W. Bodanko (Częstochowa)
}

Introduction. Dans 'la présente note nous démontrons quelques théorèmes sur l'unicité et l'existence des solutions des problèmes de Fourier dans des domaines non bolnés, et du problème de Cauchy, pour l'équation linéaire du type parabolique

(1) $\quad F(u) \equiv \sum_{i, j=1}^{n} a_{i j}(x, t) u_{x i x j}^{\prime \prime}+\sum_{i=1}^{n} b_{i}(x, t) u_{x_{i}}^{\prime}+c(x, t) u-u_{i}^{\prime}=f(x, t)$

et pour le système du type parabolique

(2) $\quad \frac{\partial u_{s}}{\partial t}=F_{s}\left(x, t, u_{i}, \frac{\partial u_{s}}{\partial x_{j}}, \frac{\partial^{2} u_{g}}{\partial x_{j} \partial x_{k}}\right) \quad(s, i=1,2, \ldots, m ; j, k=1,2, \ldots, n)$.

Nous y étudions, de plus, certaines inégalités différentielles aux dérivées partielles du type parabolique et nous démontrons des théorèmes analogues à [1] et [2].

M. Krzyżanski (voir [5]) a démontré que si les coefficients de l'équation (1) satisfont aux conditions suivantes

$$
\left|a_{\imath \jmath}(x, t)\right| \leqslant M, \quad\left|b_{\imath}(x, t)\right| \leqslant M\left(r^{2}+1\right)^{1 / 2}, \quad c(x, t) \leqslant M\left(r^{2}+1\right)
$$

où

$$
r^{2}=\sum_{i=1}^{n} x_{i}^{2} \quad(i, j=1,2, \ldots, n),
$$

alors le premier problème de Fourier (FI) admet dans la classe $E_{2}$ (voir § 1 ) une solution au plus.

Ce dernier théorème s'étend au système (2) (voir [1]).

Dans [3] on a démontré l'unicité de la solution du problème de Cauchy dans la classe des fonctions bornées si les coefficients de l'équation (1) remplissent les conditions suivantes:

$$
\left|a_{\imath \jmath}(x, t)\right| \leqslant M\left(r^{2}+1\right), \quad\left|b_{\imath}(x, t)\right| \leqslant M\left(r^{2}+1\right)^{1 / 2}, \quad c(x, t) \leqslant M .
$$


Dans le présent travail nous allons démontrer un théorème sur l'unicité du problème (FI) dans le classe $E_{2 a}(0 \leqslant a<\infty)$, si les coefficients de l'équation (1) satisfont aux inégalités

(5) $\left|a_{i j}(x, t)\right| \leqslant M\left(r^{2}+1\right)^{1-a}, \quad\left|b_{i}(x, t)\right| \leqslant M\left(r^{2}+1\right)^{1 / 2}, \quad c(x, t) \leqslant M\left(r^{2}+1\right)^{a}$

$$
(i, j=1,2, \ldots, n) \text {. }
$$

Ce théorème s'étend aux solutions des second et troisième problèmes de Fourier (FI-FIII) relatifs à l'équation (1) et (2).

J'exprimemes sincères remerciements à prof. M. Krzyżański qui a bien voulu m'aider dans la rédaction de ce travail.

§ 1. Définitions et théorèmes auxillaires. Soit $(x, t)$ un point variable $\left(x_{1}, \ldots, x_{n}, t\right)$ de l'espace euclidien $\grave{a}(n+1)$ dimensions $E_{n+1}$. Soient encore:

$\Omega$ - un domaine non borné dans l'espace $t_{n}$ des variables $\left(x_{1}, \ldots, x_{n}\right)$,

$D=\Omega \times(0, T)$ le produit topologique de $\Omega$ et $(0, T)$,

$\sigma=\mathrm{F}(\Omega) \times(0, T]$, où $\mathrm{F}(\Omega)$ est la frontière de $\Omega$,

$D^{T_{1}}=\left\{(x, t):(\infty, t) \in D, t \leqslant T_{1} \leqslant T\right\}$.

Nous supposons que les coefficients de l'équation (1) et la fonction $f(x, t)$ sont définies dans $D$ et que la forme

est définie positive.

$$
\sum_{i, j=1}^{n} a_{i j}(x, t) \lambda_{i} \lambda_{j}
$$

Les fonctions $F_{s}\left(x, t, y_{i}, z_{j}, z_{j k}\right)(s, i=1,2, \ldots, m ; j, k=1,2, \ldots, n)$ sont définies pour $(x, t) \in D$ et $y_{i}, z_{j}, z_{j k}$ arbitraires.

DÉFINIrron 1. Nous dirons qu'une fonction $F^{\prime}(x, t)$ est de classe $L_{a}^{\prime}$ $(a>0)$ dans un domaine $D$, s'il existe un nombre positif $A$ tel que

$$
|F(x, t)| \leqslant \exp \left\{A\left(r^{2}+1\right)^{a / 2}\right\} \quad \text { pour } \quad(x, t) \in D \text { et } r^{2}=\sum_{i=1}^{n} x_{i}^{2} .
$$

Remarque 1 . La classe $E_{0}$ est la classe des fonctions satisfaisant aux inégalités

$$
|u(x, t)| \leqslant M\left(r^{2}+1\right)^{d} \quad \text { et } \quad 0 \leqslant \delta<1 .
$$

Lemare 1. Nous supposons que les coefficients de l'équation (1) satisfont aux conditions (5) dans $D$ et que $u(x, t)$ est une fonction de classe $\mathbb{H}_{2 a}$ $(0 \leqslant a<\infty)$ dans $D$.

Alors il existe une fonction $H(x, t, K, \beta)$ définie et positive dans $D$ telle que

$$
\begin{gathered}
\lim _{|x| \rightarrow \infty}(u(x, t) / H(x, t, K, \beta))=0 \quad \text { uniformément pour } \quad t \in(0, T), \\
F(H) / H \leqslant-1 \quad \text { pour } \quad(x, t) \in D^{T_{1}},
\end{gathered}
$$

a constante $T_{1}$ dependant de $a, M$ et $n$. 
Démonstration. Nous posons

$$
H(x, t, K, \beta)=\exp \left\{K\left(r^{2}+1\right)^{a} e^{\beta t}\right\} \quad \text { pour } \quad 0<a<\infty ;
$$

On peut choisir le nombre $K>1$ de sorte que l'on ait (6). On a.

$$
\begin{aligned}
F(H) / H= & e^{\theta t} K\left[2 \alpha\left(r^{2}+1\right)^{(a-1)} \sum_{i=1}^{n} a_{i i}+e^{\beta t} 4 K \alpha^{2}\left(r^{2}+1\right)^{2(\alpha-1)} \sum_{i, j=1}^{n} a_{i j} x_{i} x_{j}+\right. \\
& +4 \alpha(\alpha-1)\left(r^{2}+1\right)^{(a-2)} \sum_{i, j=1}^{n} a_{i j} x_{i} x_{j}+2 \alpha\left(r^{2}+1\right)^{(a-1)} \sum_{i=1}^{n} b_{i} x_{i}+ \\
& \left.+c e^{-\beta t} / K-\beta\left(r^{2}+1\right)^{a}\right] .
\end{aligned}
$$

Nous choisissons une constante $M_{1}$ dépendant de $a, M$ et $n$ de façon que

(a)

$$
\left|2 a\left(r^{2}+1\right)^{(a-1)} \sum_{i=1}^{n} a_{i n}\right| \leqslant M_{1}
$$

$$
\left|e^{\beta t} 4 K \alpha^{2}\left(r^{2}+1\right)^{2(a-1)} \sum_{i, j=1}^{n} a_{i j} x_{i} x_{j}\right| \leqslant M M_{1}\left(r^{2}+1\right)^{a} K e^{\beta t}
$$

(c)

$$
\begin{gathered}
\left|4 \alpha(\alpha-1)\left(r^{2}+1\right)^{(a-2)} \sum_{i, j=1}^{n} a_{i j} x_{i} x_{j}\right| \leqslant M_{1}, \\
\left|2 \alpha\left(r^{2}+1\right)^{(a-1)} \sum_{i=1}^{n} b_{i} x_{i}\right| \leqslant M_{1}\left(r^{2}+1\right)^{a} .
\end{gathered}
$$

Enfin nous obtenons l'inégalité

$$
F(H) / H \leqslant e^{\beta t} K\left(r^{2}+1\right)^{a}\left(M_{2} K e^{\beta t}-\beta\right) \leqslant-1
$$

pour $\beta=M_{2} K e+1$ et $(x, t) \in D^{T_{1}}, T_{1}=1 / \beta$.

Dans le cas où $\alpha=0$ nous posons

Alors

$$
H(x, t, K, \beta)=\left(r^{2}+1\right) e^{\beta t} \text {. }
$$

$$
\begin{aligned}
F(H) / H & =\left[2 \sum_{i=1}^{n} a_{i i}+2 \sum_{i=1}^{n} b_{i} x_{i}+c\left(r^{2}+1\right)-\beta\left(r^{2}+1\right)\right] /\left(r^{2}+1\right) \\
& \leqslant\left[M M_{1}\left(r^{2}+1\right)-\beta\left(r^{2}+1\right)\right] /\left(r^{2}+1\right) \leqslant-1
\end{aligned}
$$

pour $\beta=M_{1}+1$ et $(x, t) \in D$.

LEMME 2. Soit $H(x, t, K, \beta)$ la fonction introduite dans le Lemme 1. On a

$\frac{H(x, t, K, \beta(K))}{H(x, t, K+k, \beta(K+k))} \leqslant \exp \left\{-k\left(r^{2}+1\right)^{a}\right\} \quad$ pour $\quad k>0$ et $0<a<\infty$. 
Démonstration. On a

$$
\begin{aligned}
\frac{H(o, t, K, \beta(K))}{H(\alpha, t, K+k, \beta(K+k))} & =\exp \left\{K\left(r^{2}+1\right)^{a} e^{\beta(K) t}-(K+k)\left(r^{2}+1\right)^{a} e^{\beta(K+k) t}\right\} \\
& \leqslant \exp \left\{-k\left(r^{2}+1\right)^{a}\right\}
\end{aligned}
$$

puisque $\beta(K) \leqslant \beta(K+k)$ pour $k>0$.

Défrintruon 2. Nous dirons qu'un domaine $D$ satisfait à la condition $\left(G_{m}\right)$, lorsque

1. $D=\Omega \times(0, T)$ et la frontière $\mathrm{F}(\Omega)$ est bornée.

2. La frontière $F(\Omega)$ est par hypothèse une surface dont l'équation s'écrit sous la forme

$$
G\left(x_{1}, \ldots, x_{n}\right)=0
$$

$G(x)$ étant une fonction de classe $O^{2}$ et ne s'annulant pas dans le domaine $\Omega$, dont les dérivées secondes sont bornées dans ce domaine, et de classe $\sigma^{1}$ dans la fermeture $\bar{\Omega} \mathrm{du}$ domaine $\Omega$. Nous supposons que $G(x)=r$ pour $r>R_{0}, R_{0}$ étant un nombre positif.

3. A chaque point $(x, t)$ de $\sigma$ corresporident des demi-droites $l_{s}$ $(s=1, \ldots, m)$ pénétrant à l'intérieur de $D$, parallèles au plan $t=0$, telles que

$$
\cos \left(l_{s}, n\right) \geqslant \varrho_{0}>0 \quad \text { pour } \quad(x, t) \in \sigma, \quad s=1, \ldots, m .
$$

Dans le cas où $m=1$, à chaque point $(x, t) \in \sigma$ correspond une seule droite pénétrant à l'intérieur de $D$ et nous la désignerons par $l$.

4. $\operatorname{grad}^{2} G(x)=\sum_{i=1}^{n}\left(G_{x_{1}}^{\prime}\right)^{2} \geqslant g_{0}^{2}>0, g_{0}$ étant un nombre constant.

Nous démontrons d'abord le lemme suivant:

LEMME 3. Nous supposons que

1. le domaine $D$ satisfait à la condition $\left(\mathrm{G}_{1}\right)$.

2. les coefficients de l'équation (1) satisfont aux conditions (5) pou' $(\infty, t) \in D$,

3. $u(x, t)$ est une fonction de classe $E_{2 a}(0 \leqslant a<\infty)$ dans le domaine $D$,

4. la fonction $h(x, t)$ est définie et bornée supérieurement pour $(x, t) \in \sigma$.

Dans toutes ces hypothèses il existe une fonction $H(x, t, K, \beta, v)$, definie et positive dans le domaine $D$, telle que

(8) $\lim _{|x| \rightarrow \infty}(u(x, t) / H(x, t, K, \beta, v))=0$ uniformément pour $t \epsilon(0, T)$,

(9) $\quad E^{\prime}(H) / H \leqslant-1 \quad$ pour $\quad(x, t) \in D^{T_{1}} \quad\left(T_{1} \leqslant T^{\prime}\right)$,

(10) $L(H) / H \leqslant-1$ pour $(x, t) \in \sigma$, 
où

$$
L(W)=\frac{d W}{d l}+h(x, t) W
$$

la constante $T_{1}$ dépendant de a, $M$ et $n$.

Démonstration. Soit $0<a<\infty$. Nous posons

$$
H(\alpha, t, K, \beta, v)=\exp \left\{K\left[(G+1)^{a}-p\right]^{2} e^{\beta t}+v t\right\}
$$

On peut supposer que $G(x)>0$ dans $\Omega$ et $K, p>1, \beta, \nu>0$.

Nous choissisons le nombre $K$ de sorte que l'on ait (8). Comine

$$
G_{x i}^{\prime}=\cos \left(\infty_{i}, n\right)\left[\sum_{j=1}^{n}\left(G_{x j}^{\prime}\right)\right]^{1 / 2}=\cos \left(x_{i}, n\right)|\operatorname{grad} G|
$$

on a

$$
\begin{aligned}
\underset{(x, t) \in \sigma}{L(H) / H} & =2 K a e^{\beta t}(1-p) \sum_{i=1}^{n} G_{x_{i}}^{\prime} \cos \left(l, x_{i}\right)+h(x, t) \\
& =2 K a \theta^{\beta t}(1-p)|\operatorname{grad} G| \cos (l, n)+h(x, t) \\
& \leqslant 2 a g_{0} \varrho_{0}(1-p)+h_{0}
\end{aligned}
$$

où $h_{0}=\max h(x, t)$ pour $(x, t) \in \sigma$.

On peut donc choisir le nombre $p$ de façon que l'on ait

$$
L(H) / H \leqslant-1 \quad \text { pour } \quad(x, t) \in \sigma ;
$$

$$
\begin{aligned}
& F^{\prime}(H) / H=K e^{\beta t}\left\{e^{\beta t} 4 K \alpha^{2}\left[(G+1)^{a}-p\right]^{2}(G+1)^{2(a-1)} \sum_{i, j=1}^{n} a_{i j} G_{x i}^{\prime} G_{x_{j}}^{\prime}+\right. \\
&+2 a^{2}(G+1)^{2(a-1)} \sum_{i, j=1}^{n_{i}} a_{i j} G_{x_{i}}^{\prime} G_{x_{j}}^{\prime}+ \\
&+2 a(a-1)\left[(G+1)^{a}-p\right](G+1)^{(a-2)} \sum_{i, j=1}^{n} a_{i j} G_{x_{i}}^{\prime} G_{x_{j}}^{\prime}+ \\
&+2 \alpha\left[(G+1)^{a}-p\right](G+1)^{(a-1)} \sum_{i, j=1}^{n} a_{i j} G_{x_{i x j}}^{\prime \prime}+ \\
&+2 \alpha\left[(G+1)^{a}-p\right](G+1)^{(a-1)} \sum_{i=1}^{n} b_{i} G_{x_{i}}^{\prime}+ \\
&\left.+c / K e^{\beta t}-\beta\left[(G+1)^{a}-p\right]^{2}\right\}-v
\end{aligned}
$$


En vertu des hypothèses faites sur la fonction $G(x)$, il existe des constantes positives $M_{\mathrm{J}}$ et $M_{2}$ telles que

$$
\begin{aligned}
M_{2}\left(r^{2}+1\right)^{a} \leqslant & {\left[(G+1)^{a}-p\right]^{2} \leqslant M_{1}\left(r^{2}+1\right)^{a}, } \\
& \left|(G+1)^{2(a-1)}\right| \leqslant M_{1}\left(r^{2}+1\right)^{a-1}, \\
& \left|(G+1)^{a}-p\right| \leqslant M_{1}\left(r^{2}+1\right)^{a / 2}, \\
& \left|(G+1)^{(a-2)}\right| \leqslant M_{1}\left(r^{2}+1\right)^{a / 2-1}, \\
& \left|(G+1)^{(a-1)}\right| \leqslant M_{1}\left(r^{2}+1\right)^{a / 2-1 / 2}
\end{aligned}
$$

pour $r \geqslant R_{1}=R_{1}\left(R_{0}, p\right) \geqslant R_{0}$.

Par conséquent nous obtenons

$$
\left|\alpha^{2} \sum_{i, j=1}^{n} a_{i j}\left[(G+1)^{\alpha}-p\right]^{2}(G+1)^{2(\alpha-1)} G_{x i}^{\prime} G_{x j}^{\prime}\right| \leqslant M_{\mathrm{a}}\left(r^{2}+1\right)^{\alpha},
$$

$$
\left|\alpha^{2} \sum_{i, j=1}^{n} a_{i j}(G+1)^{2(a-1)} G_{x_{i}}^{\prime} G_{x j}^{\prime}\right| \leqslant M_{\mathrm{a}}
$$

$$
\left|\alpha(\alpha-1) \cdot \sum_{i, j=1}^{n} a_{i j}\left[(G+1)^{a}-p\right](G-1)^{(\alpha-2)} G_{x_{i}}^{\prime} G_{x_{1}}^{\prime}\right| \leqslant M_{3} .
$$

$$
\left|\alpha \sum_{i, j=1}^{n} a_{i j}\left[(G+1)^{a}-p\right](G+1)^{(a-1)} G_{x i, j}^{\prime \prime}\right| \leqslant M_{3}
$$

$$
\left|\alpha \sum_{i, j=1}^{n} b_{i}\left[(G+1)^{a}-p\right](G+1)^{(a-1)} G_{i i}^{\prime}\right| \leqslant M_{\mathbf{3}}\left(\gamma^{2}+1\right)^{a} .
$$

Enfin, pour $r \geqslant R_{1}$, on a

si

$$
F^{\prime}(H) / H \leqslant K e^{\beta t}\left(r^{2}+1\right)^{a}\left(K e^{\beta t} M \Gamma_{4}-\beta M \Gamma_{2}\right)-v \leqslant-1
$$

$$
\beta=\left(M_{1} K e+1\right) / M_{2}, \quad t \leqslant T_{1}=1 / \beta \quad \text { et } \quad \nu \geqslant 0 .
$$

Pour $r \leqslant R_{1}$ les fonctions $a_{i j}, b_{i}, c, G, G_{x_{i}}^{\prime}, G_{x_{i j j}}^{\prime \prime}$ sont bornées; on peut donc choisir le nombre $v$ de façon que l'on ait l'inégalité (9).

Pour $\alpha=0$ nous posons

$H(x, t, K, \beta)=K(G(x) p-1)^{2} e^{\beta t}+\beta t+1, \quad K>1, p>0, \beta>0$.

Donc

$$
\begin{aligned}
\underset{(x, t) \sigma \sigma}{L(H) / H} & =\frac{-2 K p e^{\beta t} \sum_{i=1}^{n} G_{i \mathfrak{i} i}^{\prime} \cos \left(l, x_{i}\right)}{K e^{\beta t}+\beta t+1}+-h(x, t) \\
& \leqslant \frac{-2 p g_{0} \varrho_{0} K e^{\beta t}+\left(K e^{\beta t}+\beta t+1\right) h_{0}}{K e^{\beta t}+\beta t+1}=-\frac{-2 p g_{0} \varrho_{0}+k(t) h_{0}}{k(t)}
\end{aligned}
$$

où $h_{0}=\sup h(x, t)$ pour $(x, t) \in \sigma$ et $k(t)=1+\beta t / K e^{\beta t}+1 / K e^{\beta t}$. 
Comme la fonction $k(t)$ est bornée pour $K>1, t, \beta>0$, il existe un nombre $p$ tel que

$$
L(H) / H \leqslant-1 \quad \text { pour } \quad K>1, t, \beta>0 \text { arbitraires. }
$$

D'apiès les hypothèses sur la fonction $G(\mathfrak{m})$ on a

$$
\begin{gathered}
\bar{M}_{1}\left(r^{2}+1\right) \leqslant(G(x) p-1)^{2} \leqslant M_{1}\left(r^{2}+1\right), \\
|G(x) p-1| \leqslant M_{1}\left(r^{2}+1\right)^{1 / 2}, \\
\left|G_{x_{1}}^{\prime}\right| \leqslant M_{1}, \quad\left|G_{x_{1} x_{1}}^{\prime \prime}\right| \leqslant M_{1}\left(r^{2}+1\right)^{-1 / 2} \text { pour } r \geqslant R_{2}=R_{2}\left(R_{0}, p\right) \geqslant R_{0},
\end{gathered}
$$

et par conséquent

$$
\begin{gathered}
\left|\sum_{i, j=1}^{n} a_{i j} G_{x_{i}}^{\prime} G_{x j}^{\prime}\right| \leqslant M_{2}\left(r^{2}+1\right), \\
\left|\sum_{i, j=1}^{n} a_{i j}(G(x) p-1) G_{x_{i} x_{j}}^{\prime \prime}\right| \leqslant M_{2}\left(r^{2}+1\right), \\
\left|\sum_{i=1}^{n} b_{i}(G(x) p-1) G_{x_{i}}^{\prime}\right| \leqslant M_{2}\left(r^{2}+1\right) .
\end{gathered}
$$

Posons

$$
M_{3}=\max \left(p n^{2} M_{2}, M M_{1} / 2 p, \sup M(\beta t+1) / 2 K p e^{\beta t}\right)
$$

on a

$$
\begin{aligned}
F(H) / H & \leqslant \frac{2 K p e^{\beta t}\left(r^{2}+1\right)\left(M_{3}-\beta \bar{M}_{1} / 2 p\right)-\beta}{H} \\
& \leqslant \frac{2 K p e^{\beta t}\left(r^{2}+1\right)\left(M_{3}-\beta \bar{M}_{1} / 2 p\right)-\beta}{K M_{1}\left(r^{2}+1\right) e^{\beta t}+\beta t+1}
\end{aligned}
$$

pour $\beta \bar{M}_{1} / 2 p>M_{3}$ et $r \geqslant R_{2}$. Donc

$$
F(H) / H \leqslant-1 \quad \text { pour } \quad \beta=\beta_{1}, r \geqslant R_{2} \text { et } t \beta_{1} \leqslant 1
$$

(le nombre $K=K(p)>1$ est choisi de façon que l'on ait (8)).

Si $r<R_{2}$ les fonctions $a_{i j}, b_{i}, c, G, G_{x l}^{\prime}, G_{x_{i} x j}^{\prime \prime}$ sont bornées et il existe un $\beta=\beta_{2}$ tel que

$$
F(H) / H \leqslant-1 \quad \text { pour } \quad r \leqslant R_{2} \text { et } \beta_{2} t \leqslant 1 .
$$

Enfin, pour $\beta_{3}=\max \left(\beta_{1}, \beta_{2}\right)$ nous avons l'inégalité

$$
F(H) / H \leqslant-1 \quad \text { pour } \quad(x, t) \in D^{T_{1}} \text { et } T_{1}=1 / \beta_{3} .
$$

Remarque 2. La constante $p$ dépend du domaine $D$ et de la fonction $h(x, t)$. 
LAMME 4. Soit $H(x, t, K, \beta(K), \nu(K))$ la fonction definie dans Lemme 3.

On a

$$
\frac{H(x, t, K, \beta(K), \nu(K))}{H(x, t, K+k, \beta(K+k), \nu(K+k))} \leqslant \exp \left\{-k\left[(G+1)^{a}-p\right]^{2}\right\}
$$

pour $k>0$ et $0<\alpha<\infty$.

La démonstration du Lemme 4 est analogue à celle du Lemme 2.

\section{§ 2. Théorèmes sur l'unicité de la solution des problèmes de Fourier.}

Defrinition 3. Nous disons que la fonction $\varphi(x, t)$ est régulière dans $\bar{D}$ si elle admet des derivées $\varphi_{x_{\ell}}^{\prime}, \varphi_{x_{1} x_{j}}^{\prime \prime}, \varphi_{l}^{\prime}(i, j=1, \ldots, n)$ continues dans $D$ et si elle est continue dans $\bar{D}$.

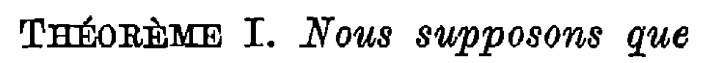

1. la fonotion $u(x, t)$ est une solution de l'équation (1) régulière et de classe $E_{2 a}$ dans $\bar{D}(0 \leqslant \alpha<\infty)$,

2. $f(x, t) \leqslant 0$ dans $D(f(x, t) \geqslant 0$ dans $D)$,

3. $u(x, t) \geqslant 0$ pour $(x, t) \in \Gamma=\bar{\Omega} \cup \sigma(u(x, t) \leqslant 0$ sur $\Gamma)$,

4. les coefficients de l'équation (1) satisfont aux conditions (5) dans $D$.

Alors $u(x, t) \geqslant 0$ dans $D(u(x, t) \leqslant 0$ dans $D)$.

En particulier, sous les hypotheses 1 et 4 , si $f(x, t)=0$ dans $D$ et $u(x, t)=0$ sur $\Gamma$, alors $u(x, t)=0$ dans $D$.

La démonstration est pareille à celle du Théorème 1 de [5].

Désignons par $D_{R}$ l'ensemble ouvert séparé de $D$ par la surface $W_{R}=\left\{(x, t): \sum_{i=1}^{n} x_{i}^{2}=R^{2}\right\}$, par $\Omega_{R}$ et $\sigma_{R}$ les parties des ensembles $\Omega$ et $\sigma$ situées à l'intérieur de $W_{R}$ et sur $W_{R}$, enfin par $C_{R}$ la partie de $W_{R}$ située dans $\bar{D} \cap\left\{\boldsymbol{E}_{n} \times(0, T]\right\}$.

Soit

$$
\Sigma_{R}=\sigma_{R} \cup C_{R}, \quad \Gamma_{R}=\bar{\Omega}_{R} \cup \Sigma_{R}
$$

et $H(x, t, K, \beta)$ une fonction positive dans $D$ et satisfaisant à la condition (6)-(7).

La fonction $v(x, t)=u(x, t) / H$ satisfait dans le domuine $D^{T_{\iota}}$ à l'équation

$$
\bar{F}(v) \equiv \sum_{i, j=1}^{n} a_{i j} v_{x_{i} x_{j}}^{\prime \prime}+\sum_{i=1}^{n} \bar{b}_{i} v_{x_{i}}^{\prime}+\bar{c} v-v_{t}^{\prime}=\bar{f}(x, t),
$$

où l'on a $\bar{c}=F(H) / H \leqslant-1$ et $\bar{f}=f / H \leqslant 0$. 
Soit $(\bar{x}, \bar{t})$ un point quelconque de $D^{T_{1}}$ et $\varepsilon>0$ un nombre arbitraire. Nous choisissons un nombre $R_{0}>0$ de façon que l'on ait:

$$
|v(x, t)|<\varepsilon \quad \text { pour } \quad r \geqslant R_{0} \text { et }(\bar{x}, \bar{t}) \in D_{R_{0}}^{T_{1}} .
$$

D'après le principe de l'extremum on a $v(\bar{x}, \bar{t}) \geqslant-\varepsilon$ puisque $v(x, t)>$ $>-\varepsilon \operatorname{pour}(x, t) \in \Gamma_{R_{0}}^{T_{1}}$.

Le nombre $\varepsilon$ étant arbitraire, on a $v(\bar{x}, \bar{t}) \geqslant 0$.

Nous avons ainsi démontré que $u(x, t) \geqslant 0$ dans $D^{T_{1}}$.

On divise ensuite le domaine $D$ en domaines partiels par les plans $t=T_{1} n \quad(n=1, \ldots, r)$ et on établit de proche en proche l'inégalité $u(x, t) \geqslant 0$ dans ces domaines.

THínìme II. Nous supposons que les hypothèses 1, 2, 4 du Théorème I sont satisfaites et que

$$
c(x, t) \leqslant 0, \quad u(x, t) \geqslant-M \text { sur } \Gamma \quad(u(x, t) \leqslant M \text { sur } \Gamma),
$$

où $M$ èst un nombre non négatif on a aussi $u(x, t) \geqslant-M$ dans $D$ $(u(x, t) \leqslant M$ dans $D)$.

Pour la démonstration on pose

$$
\bar{u}(x, t)=u(\dot{x}, t) \pm M
$$

et on applique le Théorème $\mathrm{I}$.

THÉORÈME III. Nous supposons que

1. le domaine $D$ satisfait d la condition $\left(\mathrm{G}_{1}\right)$,

2. la fonction $u(x, t)$ est une solution de l'équation (1), régulière et de classe $E_{2 a}$ dans $\bar{D}$,

3. il existe une dérivée du/dl pour $(x, t) \in \sigma$ et

$$
L(u) \equiv \frac{d u}{d l}+h(x, t) u \leqslant 0 \quad \text { sur } \sigma \quad(L(u) \geqslant 0),
$$

la fonction $h(x, t)$ est bornée supérieurement pour $(x, t) \in \sigma$,

4. $u(x, 0) \geqslant 0$ pour $x \in \Omega(u(x, 0) \leqslant 0$ pour $x \in \Omega)$,

5. les coefficients de l'équation (1) satisfont aux conditions (5),

6. $f(x, t) \leqslant 0$ dans $D(f(x, t) \geqslant 0$ dans $D)$.

Alors $u(x, t) \geqslant 0$ pour $(x, t) \in D(u(x, t) \leqslant 0$ pour $(x, t) \in D)$.

En particulier $u(x, t)=0$ si $f(x, t)=0$ dans $D, L(u)=0$ sur $\sigma$ et $u(x, 0)=0$ dans $\Omega$.

Démonstration. Soit $H(x, t, K, \beta, \nu)$ une fonction positive dans $D$ et choisie de façon que l'on ait les relations (8)-(10) (voir Lemme 3).

La fonction $v(x, t)=u(x, t) / H$ satisfait dans $D^{T_{1}}$ à l'équation (14) où l'on a

$$
\bar{c}=F(H) / H \leqslant-1 \quad \text { et } \quad \bar{f}=f / H \leqslant 0 .
$$


Ensuite on procède de même manière que dans la démonstration du Théorème dans [6].

DÉfINITION 4. La fonction $F_{s}\left(x, t, y_{i}, z_{j}, z_{j k}\right) \quad(i=1,2, \ldots, m ; j, k$ $=1,2, \ldots, n)$ est dite elliptique par rapport à un système de fonctions

$$
w_{1}(x, t), \ldots, w_{m}(x, t)
$$

de clasise $C^{1}$ dans $D$, lorsque

$$
F_{s}\left(x, t, w_{i}, \frac{\partial w_{s}}{\partial x_{j}}, z_{j k}\right)-F_{s}\left(x, t, w_{i}, \frac{\partial w_{s}}{\partial x_{j}}, \bar{z}_{j k}\right) \leqslant 0 \quad \text { pour } \quad(x, t) \in D
$$

pour tout système de nombres $z_{j k}=z_{k j}, \bar{z}_{j k}=\bar{z}_{k j}(j, k=1, \ldots, n)$ tel que la forme quadratique

est non positive.

$$
\sum_{j, k=1}^{n}\left(z_{j k}-\bar{z}_{j k}\right) \lambda_{i} \lambda_{j}
$$

Dífinition 5: Nous disons que les fonctions $F_{s}\left(x, t, y_{i}, z_{j}, z_{j k}\right)$ $(s, i=1, \ldots, m, j, k=1, \ldots, n)$ satisfont à la condition $\left(\mathrm{L}_{a}\right)(0 \leqslant a<\infty)$ s'il existe des nombres positife $L_{1}, L_{2}, L_{3}$ tels que

$$
\begin{aligned}
& F_{s}\left(x, t, y_{i}, z_{j}, z_{j k}\right)-F_{s}\left(x, t, \bar{y}_{i}, \bar{z}_{j}, \bar{z}_{j k}\right) \\
& \leqslant L_{1}\left(r^{2}+1\right)^{(1-a)} \sum_{j, k=1}^{n}\left|z_{j k}-\bar{z}_{j k}\right|+L_{2}\left(r^{2}+1\right)^{1 / 2} \sum_{j=1}^{n}\left|z_{j}-\bar{z}_{j}\right|+L_{3}\left(r^{2}+1\right)^{\alpha} \sum_{i=1}^{m}\left|y_{i}-\bar{y}_{i}\right| \\
&(s=1, \ldots, m)
\end{aligned}
$$

pour tout système de nombres $y_{i}, \bar{y}_{i}, z_{j}, \bar{z}_{j}, z_{j k}, \bar{z}_{j k}, y_{s} \geqslant \bar{y}_{s}(j, k=1, \ldots, n$, $i=1, \ldots, m)$. En ce qui concerne le système $(2)$, nous avons le

THÉRोime IV. On suppose que les fonetions $F_{s}(s=1, \ldots, m) d u$ système (2) satisfont à condition $\left(\mathbf{L}_{a}\right)$.

Alors le premier problème de Fouriev velatif au système (2) admet dans le domaine $\bar{D}$ une solution au plus, régulière et de classe $B_{2 a}$, telle que les fonctions $F_{s}(s=1, \ldots, m)$ sont elliptique par rapport à cette solution.

THÉRonìne V. Nous supposons que

1. le domaine $D$ satisfait $\dot{a}$ la condition $\left(\mathbf{G}_{m}\right)$,

2. les fonctions $F_{s}(s=1, \ldots, m)$ satisfont $\dot{a}$ la condition $\left(\mathrm{I}_{a}\right)$,

3. les fonctions $G_{8}\left(x, t, y_{1}, \ldots, y_{m}\right)(s=1, \ldots, m)$ satisfont $\dot{a}$ la condition $(\overline{\mathrm{L}})$; il existe une constante $L$, telle que

$$
G_{s}\left(x, t, y_{1}, \ldots, y_{m}\right)-G_{s}\left(x, t, \bar{y}_{1}, \ldots, \bar{y}_{m}\right) \leqslant L \sum_{i=1}^{m}\left|y_{i}-\bar{y}_{i}\right|
$$

pour $(\infty, t) \in \sigma$ et tout système de nombres $y_{1}, \bar{y}_{i}, y_{s} \geqslant \bar{y}_{a}$. 
Alor's il existe un système au plus $u_{1}(x, t), \ldots, u_{m}(x, t)$ de fonctions de olasse $E_{2 a}$ et régulière dans $\vec{D}$, tel que

(a) $u_{1}(x, t), \ldots, u_{m}(x, t)$ est une solution du système (2),

(b) les fonctions $F_{s}(s=1, \ldots, m)$ sont elliptiques par rapport $u_{1}, \ldots, u_{m}$,

(c) $u_{i}(x, 0)=\varphi_{i}(x)$ pour $x \in \Omega \quad(i=1, \ldots, m)$ où les fonctions $\varphi_{i}(x)$ sont données et continues dans $\bar{\Omega}$,

(d) il existe des dérivées $d u_{i} / d l_{t}$ pour $(x, t) \in \sigma(i=1, \ldots, m)$ et $d u_{i} / d l_{i}+$ $+G\left(x, t, u_{1}, \ldots, u_{m}\right)=0$ pour $(x, t) \in \sigma(i=1, \ldots, m)$.

Les démonstrations des Théorèmes IV et V sont, d'apprès des Lemmes 1 et 3 analogues, à celles des théorèmes concernant le cas $a=1$ (voir [1] et [2]).

\section{liques.}

§ 3. Inégalités entre les solutions des équations parabo-

DÉfinIIION 6. Nous disons que les fonctions

$$
\psi_{8}\left(y_{1}, \ldots, y_{m}, Z\right) \quad(s=1, \ldots, m),
$$

où $Z$ ne dépend pas de $\left\{y_{\mathfrak{i}}\right\}$, satisfont à la condition (W) par rapport à $\left(y_{1}, \ldots, y_{m}\right)$ si on a l'inégalité

$$
\psi_{s}\left(y_{1}, \ldots, y_{m} ; Z\right) \leqslant \psi_{s}\left(\bar{y}_{1}, \ldots, \bar{y}_{m}, Z\right)^{\cdot} \quad(s=1, \ldots, m),
$$

pour $y_{i} \leqslant \bar{y}_{i}, i \neq s, y_{8}=\bar{y}_{8}$.

THÉRÈME VI. Nous supposons que

1. $\left\{\bar{u}_{t}(x, t)\right\},\left\{\bar{u}_{t}(x, t)\right\} \quad(i=1, \ldots, m)$ sont les solutions des systèmes

$$
\begin{aligned}
& \frac{\partial \bar{u}_{s}}{\partial t}=\bar{F}_{s}\left(x, t, \bar{u}_{i}, \frac{\partial \bar{u}_{s}}{\partial x_{j}}, \frac{\partial^{2} \bar{u}_{s}}{\partial x_{j} \partial x_{k}}\right), \\
& \frac{\partial \overline{\bar{u}}_{s}}{\partial t}=\overline{\bar{F}}_{s}\left(x, t, \overline{\bar{u}}_{i}, \frac{\partial \overline{\bar{u}}_{s}}{\partial x_{j}}, \frac{\partial^{2} \overline{\bar{u}}_{s}}{\partial x_{j} \partial x_{k}}\right)
\end{aligned} \quad(s=1, \ldots, m),
$$

régutières et de classe. $E_{2 a}$ dans $\bar{D}$,

2. pour chaque $s(s=1, \ldots, m)$ la fonction $\bar{F}_{s}$ ou $\bar{F}_{s}$ est elliptique par rapport à $\left\{\bar{u}_{i}\right\}$ ou $\left\{\overline{\bar{u}}_{i}\right\}$ respectivement),

3. pour chaque $s(s=1, \ldots, m)$ la fonction $\bar{F}_{8}$ ou $\overline{\bar{F}}_{8}$ satisfait en même temps aux conditions $\left(\mathrm{L}_{a}\right)$ et $(\mathrm{W})$, par rapport $d\left\{y_{i}\right\}$,

4. $\bar{F}_{s}\left(x, t, y_{i}, z_{j}, z_{j k}\right) \leqslant \overline{\bar{F}}_{s}\left(x, t, y_{i}, z_{j}, z_{j k}\right)(s=1, \ldots, m)$ pour $(x, t) \in D$ et $y_{i}, z_{j}, z_{j k}$ arbitraires,

5. $\bar{u}_{i}(x, t) \leqslant \overline{\bar{u}}_{i}(x, t)$ pour $(x, t) \in \Gamma(i=1, \ldots, m)$.

Alors $\bar{u}_{i}(x, t) \leqslant \overline{\bar{u}}_{i}(x, t)$ dans $D(i=1, \ldots, m)$. 
THÉRÈME VII. Nous supposons que

1. les hypothèses $1,2,3,4$ du théorème VI sont vérifiées et le domaine $D$ satisfait $\dot{a}$ la oondition $\left(\mathrm{G}_{m}\right)$,

2. $\bar{u}_{\imath}(x, 0) \leqslant \overline{\bar{u}}_{i}(x, 0)$ pour $x \in \Omega(i=1, \ldots, m)$,

3. $i l$ existe des dérivées $d \bar{u}_{\imath} / d l_{i}, d \overline{\bar{u}}_{t} / d l_{i}$ pour $(\infty, t) \in \sigma(i=1, \ldots, m)$ et

$$
\begin{aligned}
& \frac{d \bar{u}_{i}}{d l_{i}}+\bar{G}_{i}\left(x, t, \bar{u}_{1}, \ldots, \bar{u}_{m}\right)=0, \\
& \frac{d \overline{\bar{u}}_{t}}{d \bar{l}_{i}}+\overline{\bar{G}}_{i}\left(x, t, \overline{\bar{u}}_{1}, \ldots, \overline{\bar{u}}_{m}\right)=0
\end{aligned}
$$

pour $(x, t) \in \sigma$, traires,

4. $\bar{G}_{i}\left(x, t, y_{j}\right) \leqslant \overline{\bar{G}}_{l}\left(x, t, y_{j}\right) \quad(i=1, \ldots, m)$ pour $(x, t) \in \sigma$ et $y_{j}$ arbi-

5. pour chaque $s(s=1, \ldots, m)$ la fonction $\bar{G}_{s}$ ou $\overline{\bar{G}}_{s}$ satisfait conditions $(\overline{\overline{\mathrm{L}}})$ et $(\mathrm{W})$ par rapport $\left\{y_{i}\right\}$.

Alors $\bar{u}_{i}(x, t) \leqslant \overline{\bar{u}}_{t}(x, t)$ dans $D(i=1, \ldots, m)$.

Lies démonstrations des Théorèmes VI et VII sont analogues, en vertu des Lemmes 1 et 3 , à celles des théorèmes concernant le cas $a=1$ (voir [1] et [2]).

Pour l'équation (1) nous avons le

THÉRÈME VIII. Nous supposons que

1. le domaine $D$ satisfait à la condition $\left(\mathrm{G}_{1}\right)$,

2. les fonctions $u_{1}(x, t)$ et $\iota_{2}(x, t)$ sont les solutions des équations

$$
F(u)=f_{1}(x, t), \quad F(u)=f_{2}(x, t) \quad \text { respectivement, }
$$

(gulières et de classe $E_{2 a}$ dans $\bar{D}$,

3. $f_{1}(x, t) \geqslant f_{2}(x, t)$ dans $D$,

4. $u_{1}(x, 0) \leqslant u_{2}(x, 0)$ dans $\Omega$,

Б. il existe des dérivées $d u_{1} / d l, d u_{2} / d l$ et

$$
\begin{array}{ll}
\frac{d u_{1}}{d l}+G_{1}\left(x, t, u_{1}\right)=0 & \\
\frac{d u_{2}}{d l}+G_{2}\left(x, t, u_{2}\right)=0 & \text { pour } \quad(x, t) \in \sigma .
\end{array}
$$

6. $G_{1}(\infty, t, y) \leqslant G_{2}(x, t, y)$ pour $(x, t) \in \sigma$ et $y$ arbitraire,

7. la fonction $G_{1}$ ou $G_{2}$ satisfait à la condition $(\mathrm{L})$ par rapport da $y$,

8. les coefficients de l'équation (1) satisfont aux conditions (5).

Alors $u_{1}(x, t) \leqslant u_{2}(x, t)$ dans $D$. 


\section{§ 4. Existence de la solution dans un domaine non borné.} Nous admettons les hypothèses suivantes:

HYPOTHESE (H). Soit $\varphi(x, t)$ une fonction continue dans $\bar{D}$.

Pour chaque $R>R_{0}$ il existe une solution $u_{R}(\infty, t)$ de l'équation (1), régulière dans $\bar{D}_{R}$, telle que

$$
u_{R}(x, t)=\varphi(x, t) \quad \text { pour } \quad(x, t) \in \Gamma_{R} .
$$

HYPOTHÈ⿴囗 $\left(\mathrm{H}_{m}\right)$. Soit $\left\{\varphi_{i}(\infty, t)\right\}(i=1, \ldots, m)$ un système de fonctions continues dans $\vec{D}$. telle que

Pour chaque $R>R_{0}$ il existe une solution $\left\{u_{i}^{R}(x, t)\right\} \quad d u$ systeme (2)

1. les fonotions $F_{8}(s=1,2, \ldots, m)$ sont elliptiques par rapport $\dot{a}\left\{u_{i}(x, t)\right\}$,

2. $u_{i}^{R}(x, t)=\phi_{i}(x, t)$ pour $(x, t) \epsilon \Gamma_{R}$.

THÉREMME IX. Nous supposons que

1. l'hypothèse $(\mathrm{H})$ est verifiee,

2. la fonction $f(x, t)$ est de classe $E_{2 a}$ dans $D$,

3. les coefficients de l'équation (1) satisfont aux conditions (5),

4. la fonction $\varphi(x, t)$ est definie, continue et de classe $E_{2 a}$ dans $\bar{D}$,

5. $0<\alpha<\infty$.

Alors il existe une solution de l'équation (1), régulière et de classe $B_{2 \alpha}$ dans $D^{T_{1}}$, telle que

$$
u(x, t)=\varphi(x, t) \quad \text { pour } \quad(x, t) \in \Gamma^{T_{1}}
$$

le nombre $T_{1} \leqslant T$ dépendant des coefficients de l'équation (1) et des fonctions $f(x, t)$ et $\varphi(x, t)$.

La démonstration est pareille à celle du théorème de [4] concernant le cas $\alpha=1$.

Soit $\left\{R_{n}\right\}$ une suite de nombres positifs telle que $R_{n} \rightarrow \infty, R_{n+1} \geqslant R_{n}$ $\geqslant R_{0}$ et soit $\left\{u_{n}(x, t)\right\}$ une suite des solutions de l'équation (1) régulières dans $\bar{D}_{R_{n}}$ et telles que

$$
u_{n}(x, t)=\varphi(x, t) \quad \text { pour } \quad(x, t) \in \Gamma_{R_{n}} .
$$

Désignons par $H(x, t, K, \beta(K))$ une fonction positive qui satisfait aux conditions:

(a) $F^{\prime}(H) / H \leqslant-1$ pour $(x, t) \in D^{T_{1}}, T_{1} \leqslant T$,

(b) $\lim _{|x| \rightarrow \infty} f / H=0, \quad \lim _{|x| \rightarrow \infty} \varphi / H=0$ uniformément dans $(0, T)$ (voir Lemme 1).

Les fonctions $v_{n}=u_{n} / H$ satisfont dans $D_{R_{n}}^{T_{1}}$ à une équation de la forme (14) avec $\bar{f}=f / H, \bar{c}=F(H) / H \leqslant-1$. 
Soit

$$
\bar{M}=\max \left\{\sup _{D}|\varphi| H\left|, \sup _{D}\right| f|H|\right\} .
$$

Nous allons démontrer que

$$
\left|v_{n}(x, t)\right| \leqslant \bar{M} \quad \text { pour } \quad(x, t) \epsilon \overline{D_{R_{n}}^{T_{2}}}, \quad n=1,2, \ldots
$$

Supposons qu'il existe un nombre $n_{0}$ tel que

$$
\frac{\max }{D_{R_{n_{0}}}^{T_{1_{0}}}} v_{n_{0}}>\bar{M}
$$

On peut choisir un point $(\bar{x}, \bar{t}) \epsilon \overline{D_{R_{n_{0}}}^{T_{1}}}$ de sorte que

$$
v_{n_{0}}(\bar{x}, \bar{t})=\frac{\sup _{0}}{D_{R_{n_{0}}}^{T_{1}}} v_{n_{0}}(x, t)=\bar{M}+\varepsilon \quad \text { où } \quad \varepsilon>0 .
$$

D'après la définition de $\bar{M}$ on a $(\bar{x}, \bar{t}) \epsilon \Omega_{R_{n_{0}}} \times\left(0, T_{1}\right]$.

Alors

$$
-\bar{M} \leqslant \bar{f}(\bar{x}, \bar{t})=\bar{F}\left(v_{n_{0}}\right)(\bar{x}, \bar{t}) \leqslant v_{n_{0}}(\bar{x}, \bar{t}) \bar{c} \leqslant-\bar{M}-\varepsilon,
$$

ce qui est impossible. D'une manière analogue nous démontrons que $v_{n}(x, t) \geqslant-\bar{M}, n=1,2, \ldots$

Désignons $\bar{v}_{n}=u_{n} / H(x, t, K+k, \beta(K+k)), k>0$.

Les fonctions $\bar{v}_{n, m}=\bar{v}_{n}-\bar{v}_{m}$ satisfont dans $D_{R_{n, m}}^{T_{\mathrm{a}}}\left(J_{2}^{\prime} \leqslant T_{1}\right)$ (où $R_{n, m}$ $\left.=\min \left(R_{n}, R_{m}\right)\right)$ à l'équation

où

$$
\sum_{i, j=1}^{g b} a_{i j} v_{x_{i} x_{j}}^{\prime \prime}+\sum_{i=1}^{n} \overline{\overline{b_{i}}} v_{s_{i}}^{\prime}+\overline{\bar{c}} v-v_{i}^{\prime}=0
$$

$$
\bar{c}=\frac{F(H(x, t, K+k, \beta(K+k)))}{H(x, t, K+k, \beta(K+k))} \leqslant-1 \quad \text { pour } \quad(x, t) \in D^{T_{2}} .
$$

D'après le Lemme 2 et (17) on a

$$
\left|\bar{v}_{n, m}(x, t)\right| \leqslant 2 \bar{M} \exp \left\{-k\left(r^{2}+1\right)^{a}\right\} \quad \text { pour } \quad(x, t) \in D_{R_{n, m}}^{T_{2}}
$$

et, en particulier,

$$
\left|\bar{v}_{n, m}(x, t)\right| \leqslant 2 \bar{M} \exp \left\{-k\left(R_{n, m}^{2}+1\right)^{\alpha}\right\} \quad \text { pour } \quad(x, t) \in \Gamma_{R_{n, m}}^{T_{\sharp}} .
$$

En appliquant le principe de l'extremum on obtient l'inégalité $\left|\bar{v}_{n, m}(x, t)\right| \leqslant 2 \bar{M} \exp \left\{-k\left(R_{n, m}^{2}+1\right)^{a}\right\} \quad$ pour $\quad(x, t) \in D_{R_{n, m}}^{T_{\mathrm{a}}}$ et $n, m=1,2, \ldots$

Comme $u_{n}=\bar{v}_{n} H(x, t, K+k, \beta(K+k))$, la suite $\left\{u_{n}\right\}$ converge pour $n \rightarrow \infty$ vers une fonction $u(x, t)$ uniformément dans $D_{R}^{T_{\mathbf{a}}}$ pour tout $R>R_{0}$. 
La fonction $u(x, t)$ est évidemment continue dans $\bar{D}^{r_{a}}$ et de classe $E_{2 a}$ (d'après (17)).

Nous allons démontrer que la fonction $u(x, t)$ est une solution de l'équation (1) régulière dans $\bar{D}^{\boldsymbol{T}_{\mathbf{2}}}$.

Choisissons $R>R_{0}$ et soit $\vec{u}(x, t)$ une solution de l'équation (1), régulière dans $\bar{D}_{R}^{T_{2}}$, telle que

$$
\bar{u}(x, t)=u(x, t) \quad \text { pour } \quad(x, t) \in \Gamma_{R}^{T_{\mathrm{g}}} .
$$

Les fonctions $w_{n}=\bar{u}-u_{n}\left(R_{n}>R\right)$ sont régulières dans $\bar{D}_{R}^{T_{a}}$ et satisfont à l'équation $F(u)=0$ dans $D_{R}^{T_{2}}$.

Alors (voir [3], p. 10)

$$
\left|\bar{u}-u_{n}\right| \leqslant \exp \left\{\left(\sup _{D_{R}} c(x, t)\right) t\right\} \sup _{\Gamma_{R}^{T_{\Omega}}}\left|u-u_{n}\right| \quad \text { pour } \quad n>N=N(R)
$$

c'est-à-dire

$$
\bar{u}=u \quad \text { pour } \quad(x, t) \in D_{R}^{T_{\mathrm{a}}} .
$$

THÉORÈME X. Nous supposons que

1. l'hypothèse $\left(\mathrm{H}_{m}\right)$ est vérifiée,

2. les fonctions $F_{s}(x, t, 0, \ldots, 0) \quad(s=1, \ldots, m)$ sont de olasse $E_{2 \alpha}$ dans $D$,

3. les fonctions $F_{s}(s=1, \ldots, m)$ satisfont à la condition $\left(\mathrm{L}_{a}\right)$,

4. $\varphi_{s}(x, t)$ sont des fonctions définies, continues et de classe $E_{2 a}$ dans $D$,

5. $0<a<\infty$.

Alors $i l$ existe une solution $\left\{u_{\imath}(x, t)\right\}(i=1, \ldots, m)$ du système (2), régulière et de classe $E_{2 a}$ dans $\overline{D^{T_{1}}}\left(T_{1} \leqslant T\right)$ telle que $u_{i}(x, t)=p_{i}(x, t)$ pour $(x, t) \in \Gamma^{T_{1}}(i=1, \ldots, m)$.

En vertu des Lemmes 1 et 2 , la démonstration du Théorème $\mathbf{X}$ est analogue à celle du théorème concernant le cas $a=1$ (voir [1]).

Remarque 3. En appliquant les Lemmes 3 et 4 on peut démontrer des théorèmes analogues aux Théorèmes IX et $X$, relatifs aux second et troisième problèmes de Fourier.

Remarque 4. Les Théorèmes I, II, IV, VI, IX, $\mathbf{X}$ restent vrais si le domaine $D$ n'est pas cylindrique.

Remarque 5. Si $\sigma$ est un ensemble vide, nous obtenons le problème de Cauchy.

\section{Travaux cltés}

[1] P. Besala, On solutions of Fourier's first problem for a system of non-linear parabolio equations in an unbounded domain, Ann. Polon. Math. 13 (1963), p. 247-266.

[2] - Concerning solutions of an exterior boundary-value problem for a system of non-linear parabolic equalions, Ann. Polon. Math. 14 (1964), p. 289-302. 
[3] A. Ilin, A. Kंalašnikov, O. Olejnik, Équations linéaires du second ơdre du type parabolique (en russe), Успехи мат. наук 17, 3 (105), (1962), p. 3-146.

[4] M. Krzyżańki, Sur les solutions de l'equation linéaire du type parabolique, déterminéss par les oonditions initiales, Annales de la Soc. Polon. de Math. 18 (1945).

[5] - Oertaines inégalités relatives aux solutions de l'équation parabolique linéaire normale, Bull. de l'Acad. Polon. des Sci., Śrie des sci. math., astr. et phys. 7 (1959), p. 131-136.

[6] - Sur l'unioité des solutions des second et troisième problèmes de Fourier relatifs à l'équation linéaire normale du type parabolique, Ann. Polon. Math. 7 (1960), p. 201-208.

Reģu par la Rédastion le 11.2.1965 